\title{
A low-noise high dynamic-range time-domain EMI measurement system for CISPR Band E
}

\author{
C. Hoffmann ${ }^{1}$ and P. Russer ${ }^{2}$ \\ ${ }^{1}$ Technische Universität München, Lehrstuhl für Hochfrequenztechnik, Munich, Germany \\ ${ }^{2}$ Technische Universität München, Lehrstuhl für Nanoelektronik, Munich, Germany
}

\begin{abstract}
In this paper, a broadband time-domain EMI measurement system for measurements from $9 \mathrm{kHz}$ to $18 \mathrm{GHz}$ is presented that allows for compliant EMI measurements in CISPR Band E. Combining ultra-fast analogto-digital-conversion and real-time digital signal processing on a field-programmable-gate-array (FPGA) with ultrabroadband multi-stage down-conversion, scan times can be reduced by several orders of magnitude in comparison to a traditional heterodyne EMI-receiver. The ultra-low system noise floor of $6-8 \mathrm{~dB}$ and the real-time spectrogram allow for the characterisation of the time-behaviour of EMI near the noise floor. EMI measurements of electronic consumer devices and electric household appliances are presented.
\end{abstract}

\section{Introduction}

Electromagnetic interference (EMI) and electromagnetic compatibility (EMC) are traditionally measured on heterodyne EMI-receivers (Hagenhaus, 1942). The sequential measurement of typically several thousand frequencies yields long scan times on the order of hours or even days. Timedomain measurement systems can reduce scan times by several orders of magnitude. After broadband sampling of the EMI signal, the spectrum is calculated via the Fast-FourierTransform (FFT) and detectors are applied digitally. With the presented system, an EMI measurement over the complete band from $9 \mathrm{kHz}$ to $18 \mathrm{GHz}$ with an IF-bandwidth of $9 \mathrm{kHz}$ takes less than $3 \mathrm{~min}$. Using a traditional EMI-receiver with a dwell-time of $100 \mathrm{~ms}$, the measurement of roughly $2 \times 10^{6}$ frequency points would take over $55 \mathrm{~h}$.

The standardization and development of new communication technologies like WIMAX is attended by an increas-

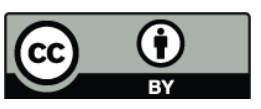

Correspondence to: C. Hoffmann (c.hoffmann@tum.de) ing usage of higher frequency bands in the high microwave range. This development implies the need for fast and comprehensive EMI measurements in the frequency range above $1 \mathrm{GHz}$.

The presented time-domain EMI measurement system allows for the CISPR-compliant measurement of EMI in CISPR Band E up to $18 \mathrm{GHz}$. The real-time capability permits the characterisation of the time-behaviour of a device's emission. Spectrogram measurements of the data transmission between two modern smartphones and the nonstationary radiated emission of a microwave oven are shown.

\section{Time-Domain EMI measurement system}

The block diagram of the presented time-domain EMI measurement system is shown in Fig. 1. The EMI-signal is received by a broadband antenna for radiated emission or a line impedance stabilization network (LISN) for conducted emission. For measurements from $9 \mathrm{kHz}$ to $1.1 \mathrm{GHz}$, the input signal is filtered by a lowpass filter to prevent spectral overlap due to a violation of Shannon's theorem. The filtered signal is sampled by the floating-point analog-to-digital converter (Braun and Russer, 2005) with a sampling rate of around $2.6 \mathrm{Gs} / \mathrm{s}$. An FPGA computes the signal spectrum via the FFT and weights the spectrum. The amplitude spectrum is displayed.

\subsection{Fast-Fourier-Transform}

To compute the EMI signal spectrum, the digitized EMIsignal is transformed by FFT. The FFT is an efficient algorithm for the computation of the Digital-Fourier-Transform (DFT). The FFT exploits symmetry and repetition properties and is defined as (Oppenheim and Schafer, 2009)

$X[k]=\sum_{n=0}^{N-1} x[n] e^{\frac{-j 2 \pi k n}{N}}$,

Published by Copernicus Publications on behalf of the URSI Landesausschuss in der Bundesrepublik Deutschland e.V. 


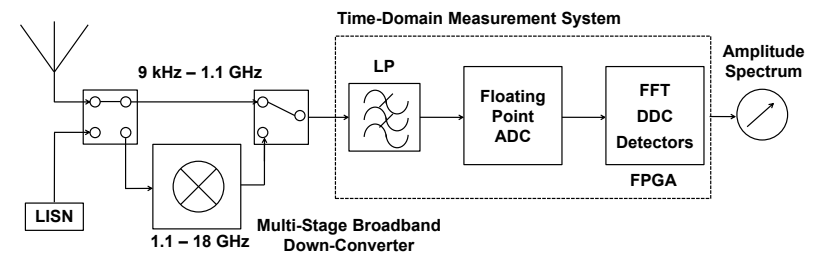

Fig. 1. Time-domain EMI measurement system.

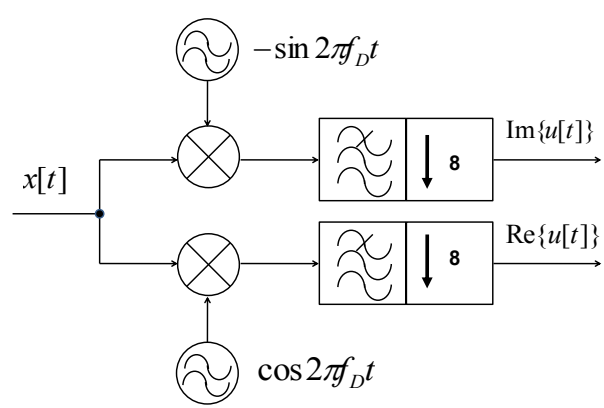

Fig. 2. Digital down-conversion, taken from Braun et al. (2009).

where $X[k]$ is the discrete amplitude spectrum of the discrete time signal $x[n]$.

\subsection{Short-Time-Fast-Fourier-Transform}

The Short-Time-Fast-Fourier-Transform (STFFT) is defined as an FFT over a limited time-interval. A Gaussian window function $w[n]$ is applied, corresponding to the IF-filter of a conventional measurement receiver. By application of the STFFT, a spectrogram is calculated. The spectrogram is a FFT taken over a time-interval of the sampled time-domain signal. It depends on the discrete time coordinate $\tau$ of the window and the discrete frequency $k$. The STFFT is calculated by (Oppenheim and Schafer, 2009)

$X[\tau, k]=\sum_{n=0}^{N-1} x[n+\tau] w[n] e^{\frac{-j 2 \pi k n}{N}}$.

\subsection{Digital Down-Conversion (DDC)}

In order to avoid data overflow in the signal processor and to enable real-time processing of the signal, the frequency range from DC to $1.1 \mathrm{GHz}$ is subdivided into eight subbands with a bandwidth of $162.5 \mathrm{MHz}$ each. For the in-phase and quadrature channel, a polyphase decimation filter reduces the sampling frequency in order to fulfill the Nyquist criterion. Every subband is digitally down-converted to the baseband and the subbands are processed sequentially (Braun and Russer, 2005). The output sampling frequency is $325 \mathrm{MHz}$, while the bandwidth is $162.5 \mathrm{MHz}$. The block diagram is shown in Fig. 2.

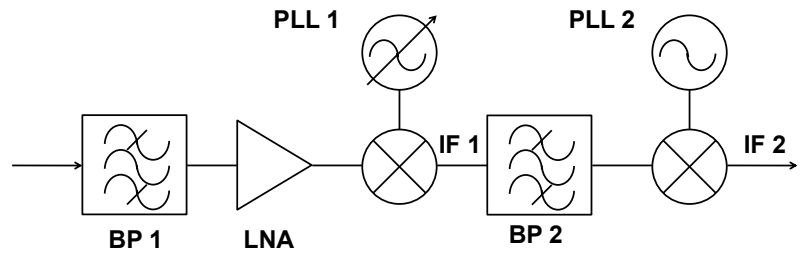

Fig. 3. $1.1-6 \mathrm{GHz}$ down-converter.

\section{Multi-stage broadband down-converter}

To overcome the limitations imposed by available analogto-digital converters, an ultra-broadband multi-stage downconverter is used to extend the frequency range up to $18 \mathrm{GHz}$. The upper frequency limit of the time-domain measurement system was increased to $3 \mathrm{GHz}$ (Braun et al., 2009), $6 \mathrm{GHz}$ (Braun et al., 2010) and $18 \mathrm{GHz}$ (Hoffmann et al., 2010) by the addition of the multi-stage broadband down-converter. The EMI signal from 1.1-6 GHz is down-converted to the frequency range below $1.1 \mathrm{GHz}$ by the $1.1-6 \mathrm{GHz}$ downconverter. The down-converted signal is sampled and the spectrum is calculated. The frequency band from $6-18 \mathrm{GHz}$ is in a first step down-converted to the input range of the $1.1-6 \mathrm{GHz}$ down-converter. In a second step, it is downconverted to the range below $1.1 \mathrm{GHz}$ and sampled.

\subsection{1-6 GHz down-converter}

The block diagram of the $1.1-6 \mathrm{GHz}$ down-converter is shown in Fig. 3. The EMI signal from $1.1-6 \mathrm{GHz}$ is downconverted by two mixer stages. The input band is divided into 16 subbands with a bandwidth of $325 \mathrm{MHz}$ each. The inherent overlap of the subbands is used to eliminate unwanted mixer spurious from the intermediate frequency band by a slight frequency shift of the subbands. The subbands are sequentially up-converted to a first intermediate frequency band IF 1 above the input band using a broadband mixer. The intermediate frequency band is filtered by the narrowband bandpass filter BP 2 and down-converted to the frequency range below $1.1 \mathrm{GHz}$ by the second mixer. The local oscillator frequencies are generated by low-noise PLL-synthesizers.

Because of the nonlinear characteristics of the mixer, a large number of mixing products are generated at its output. These frequencies $f_{\mathrm{IF}}$ are determined by (Vendelin et al., 2005)

$f_{\mathrm{IF}}^{m, \pm n}=\left|m \cdot f_{\mathrm{LO}} \pm n \cdot f_{\mathrm{RF}}\right|, m, n \in \mathbb{N}$,

where $f_{\mathrm{RF}}$ is the $\mathrm{RF}$ input frequency and $f_{\mathrm{LO}}$ is the local oscillator frequency.

If only the fundamental frequencies of $f_{\mathrm{LO}}$ and $f_{\mathrm{RF}}$ are taken into consideration, i.e. $m, n=1$, we obtain two frequency components $f_{\mathrm{RF} 1,2}$ according to Eq. (3) (Maas, 1993) 


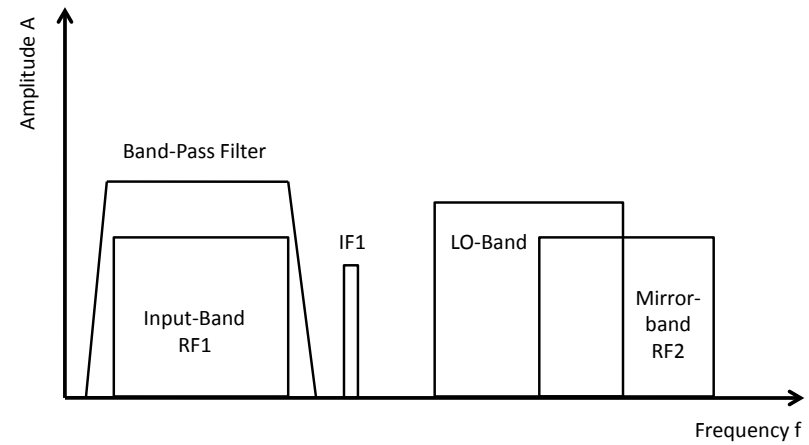

Fig. 4. Up-conversion frequency bands.

$f_{\mathrm{RF} 1,2}=\left|f_{\mathrm{LO}} \pm f_{\mathrm{IF}}\right|$

The frequency conversion yields two sidebands. The imagefrequency signal is converted to the same intermediate frequency as the desired signal. Because the input band and the image band are not overlapping spectrally, the image band is sufficiently suppressed by the preselection bandpass filter BP 1 in this two-stage mixing scheme as illustrated in Fig. 4.

The preselection band-pass filter also increases the spurious-free dynamic range of the system by suppressing low-frequency out-of-band EMI signal components. A highgain, low-noise $\mathrm{InGaP} / \mathrm{GaAs}$ MMIC preamplifier increases system sensitivity.

\section{$3.26-18 \mathrm{GHz}$ down-converter}

The block diagram of the $6-18 \mathrm{GHz}$ down-converter is shown in Fig. 5. The input band is divided into 3 ultra-wide subbands: band 1 from $6-9 \mathrm{GHz}$, band 2 from $9-13 \mathrm{GHz}$ and band 3 from 13-18 GHz. Broadband, low-insertion loss, single-input, triple-output (SP3T) PIN-diode switches are used to switch between the bands. Preselection filters increase system dynamic range. The EMI-signal is amplified by a low-noise amplifier and down-converted to the frequency band from $1.1-6 \mathrm{GHz}$ via a broadband mixer with low conversion loss. The local oscillator frequencies are generated by a low-noise PLL-Synthesizer and an active multiplier. Because the local oscillators fundamental is not sufficiently attenuated by the mixer's LO-IF isolation, a fifth order planar bandpass filter is used to suppress the fundamental by over $75 \mathrm{~dB}$.

\subsection{Noise behaviour}

With the signal-to-noise-ratios $\mathrm{SNR}_{\mathrm{in}}$ at the input and and $\mathrm{SNR}_{\text {out }}$ at the output of a system, the system's noise figure $F$ is given by (Davenport and Root, 1987)

$F=\frac{\mathrm{SNR}_{\text {out }}}{\mathrm{SNR}_{\text {in }}}$.

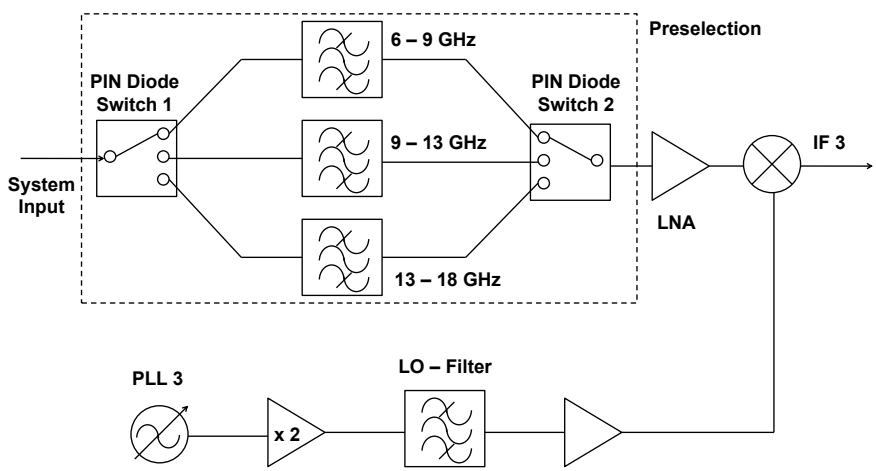

Fig. 5. 6-18 GHz GHz down-converter.

The available noise power $P_{N}$ at the output of a system with a noise figure $F$ and unity gain is defined by

$P_{N}=k T_{0} B_{\mathrm{ENB}}$,

where $k$ is the Boltzmann constant, $T_{0}$ is the ambient temperature and $B_{\mathrm{ENB}}$ is the equivalent noise bandwidth. The noise power at the output of a system, that exhibits the noise figure $F$ can be calculated by

$P_{N}=F k T_{0} B_{\mathrm{ENB}}$.

For the calculation of the system noise figure above $1 \mathrm{GHz}$, the noise figures of the multi-stage broadband downconverter components up to the first mixer have been considered. The noise figure $F$ of a cascaded system with $N$ stages is determined by

$F=1+F_{1}+\frac{F_{2}-1}{G_{1}}+\frac{F_{3}-1}{G_{1} G_{2}}+\ldots+\frac{F_{N}-1}{\prod_{k=1}^{N-1} G_{k}}$,

where $G_{i}$ is the available power gain of stage $i$ and $F_{i}$ is the noise figure of stage $i$. With Eq. (8), the system's noise figure was calculated to be less than $6 \mathrm{~dB}$ in the range from $1-6 \mathrm{GHz}$ and less than $8 \mathrm{~dB}$ from $6-18 \mathrm{GHz}$.

The theoretical average system noise floor can be calculated using Eq. (7). The equivalent noise bandwidth $B_{\mathrm{ENB}}$ of the IF-filters with Gaussian characteristic is obtained with

$B_{\mathrm{ENB}}=\int_{-\infty}^{\infty}|H(f)|^{2} d f$

using the filter's transfer functions $H(f)$. This yields an equivalent noise bandwidth $B_{\mathrm{ENB}, 9 \mathrm{kHz}}$ of $6.8 \mathrm{kHz}$ for the $9 \mathrm{kHz}$ digital IF-filter. With the $9 \mathrm{kHz}$ IF-filter we obtain a theoretical average noise floor of $-22.5 \mathrm{~dB} \mu \mathrm{V}$ from 1$6 \mathrm{GHz}$ and $-20.5 \mathrm{~dB} \mu \mathrm{V}$ from $6-18 \mathrm{GHz}$. The measured system noise floor is higher by about $2-3 \mathrm{~dB}$ though, because of cable attenuation, the minimum attenuation of the variable attenuator and mismatch effects. 


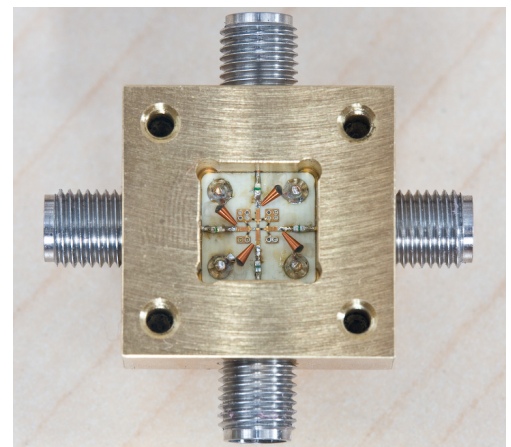

Fig. 6. SP3T PIN-diode switch.

\subsection{Dynamic range}

EMI measurement systems have to exhibit a sufficient dynamic range to be able to display transient emissions correctly. The dynamic range of a system is limited by the system noise floor on the lower end and the maximum input level without distortion of the input signal on the upper end. For spectrum analyzers, the maximum level is typically the $1 \mathrm{~dB}$ compression point $\left(P_{1 \mathrm{~dB}}\right)$ of the system. Heterodyne EMI-receivers add a preselection to increase the dynamic range by suppressing out-of-band broadband or narrowband signals and Gaussian noise.

The preselection filter with the equivalent impulse bandwidth $B_{\text {imp,pre increases the dynamic range for pulse signals }}$ by

$\Delta D_{\text {pulse }}=20 \log _{10} \frac{B_{\text {imp,sys }}}{B_{\text {imp,pre }}}$,

with the equivalent impulse bandwidth of the measurement receiver $B_{\text {imp,sys. }}$.

For Gaussian noise, the dynamic range is increased by

$\Delta D_{\text {noise }}=10 \log _{10} \frac{B_{\text {noise,sys }}}{B_{\text {noise, } \text { pre }}}$

where $B_{\text {noise,sys }}$ and $B_{\text {noise,pre }}$ are the equivalent noise bandwidths of the measurements receiver and preselection filter.

Out-of-band narrowband signals with the frequency $f_{n}$ are attenuated by the preselection filter's transfer function $H_{\text {pre }}(f)$. This yields an increase of dynamic range according to

$\Delta D_{n}=20 \log _{10} H_{\text {pre }}\left(f_{n}\right)$.

Pulse-modulated signals are used to determine the IF dynamic range of EMI measurement systems above 1 GHz. CISPR 16-1-1 (CISPR 16-1-1 Ed. 3.1 Am. 1, 2010) demands an IF dynamic range of at least $40 \mathrm{~dB}$ for EMI measurements above $1 \mathrm{GHz}$.

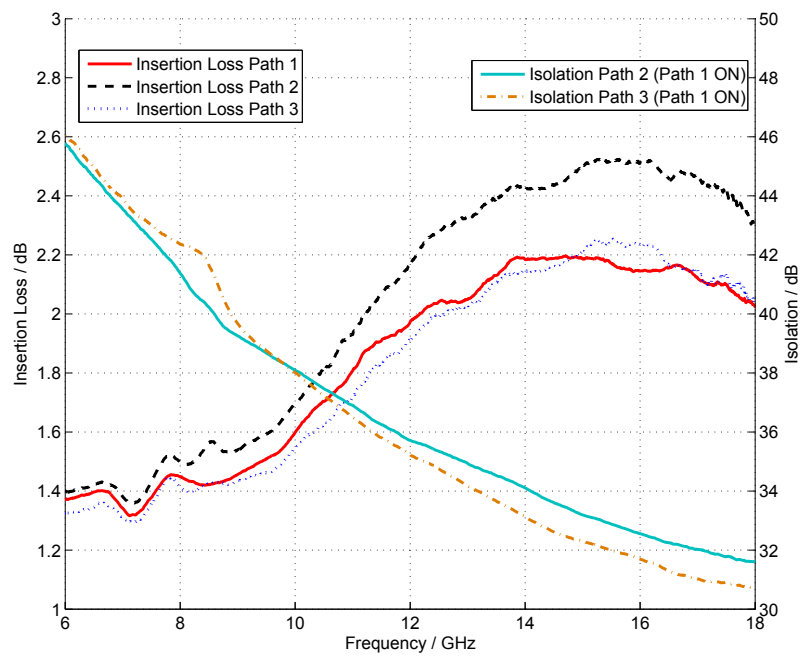

Fig. 7. Measurement of the SP3T PIN-diode switch.

\section{Hardware implementation}

As explained in Sect. 3.3, a low-noise figure of the first components in the RF Frontend is vital for maintaining a low system noise figure. PIN-diodes are commonly used in microwave and millimeter wave switches and attenuators.

By variation of the stored charge $Q=I_{F} \cdot \tau$ in the intrinsic region of a PIN-diode, its series resistance $R_{\mathrm{S}}$ can be changed by several orders of magnitude (Sze, 1988) according to

$R_{\mathrm{S}}=\frac{W^{2}}{I_{F} \cdot \tau\left(\mu_{n}+\mu_{p}\right)}$,

where $W$ is the width of the intrinsic region, $I_{F}$ is the forward bias current, $\tau$ is the minority carrier lifetime and $\mu_{n}$ and $\mu_{p}$ are the electron and hole mobilities.

PIN-diode switches with single series diodes exhibit low insertion loss in the ON-state, but cannot provide the needed isolation in the OFF-state. To increase isolation, a seriesshunt configuration was implemented, where dual shuntdiodes short-circuit the paths in the OFF-state in addition to the series diode in reverse polarity. The switches were fabricated on a glass reinforced hydrocarbon/ceramic RFsubstrate and are shown in Fig. 6.

The performance characteristics of the switches were measured with a vector network analyzer (VNA). The measured scattering parameters are shown in Fig. 7. The insertion loss for Path 1 and Path 3 is below $2.2 \mathrm{~dB}$ from $6-18 \mathrm{GHz}$. At $2.5 \mathrm{~dB}$, the insertion loss of the middle path is around $0.3 \mathrm{~dB}$ higher, because of coupling to the other two paths. The isolation exceeds $30 \mathrm{~dB}$ at $18 \mathrm{GHz}$.

\section{Measurement results}

For the characterisation of the system IF dynamic range, a pulse generator fed a pulse modulated sinusoidal signal with 


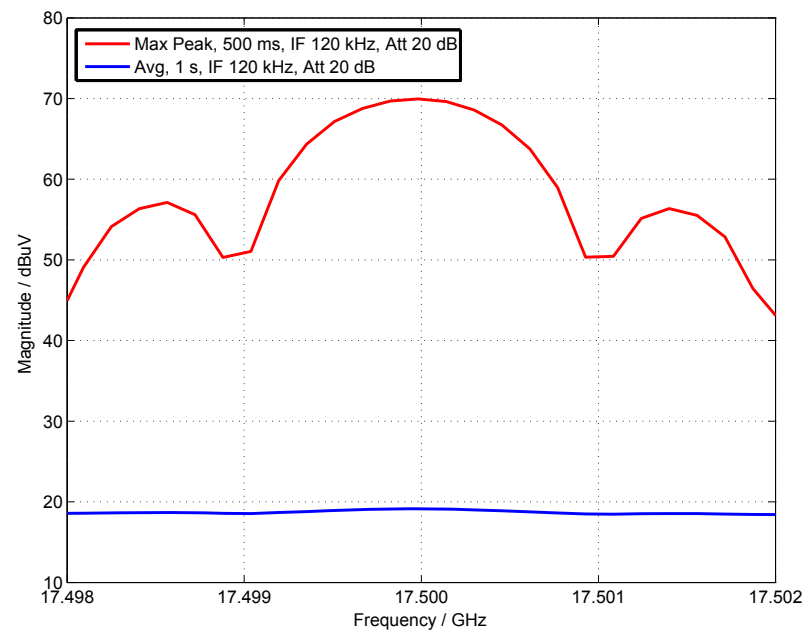

Fig. 8. Measurement of a pulse-modulated signal.

a frequency of $17.5 \mathrm{GHz}$ to the system input. The signal pulse width was set to $1 \mu$ s and the pulse period to $40 \mathrm{~ms}$. The spectrum was weighted by peak and average detectors and is shown in Fig. 8. With this pulse period, the average detector already shows the system noise floor. The difference in level between the peak and average detector measurements is defined as the IF dynamic range. The measurements indicate an IF dynamic range of over $60 \mathrm{~dB}$ for an IF-bandwidth of $1 \mathrm{MHz}$, exceeding CISPR 16-1-1 requirements by over $20 \mathrm{~dB}$.

Figure 9 shows the ultra-low system noise floor measured from $30 \mathrm{MHz}$ to $6 \mathrm{GHz}$. The system exhibits a noise floor of below $-15 \mathrm{~dB} \mu \mathrm{V}$ in this range. To quantify the noise floor above $6 \mathrm{GHz}$, the noise voltages $V_{N}$ were measured at distinct frequencies using the average detector and a matched input and the respective noise powers $P_{N}$ were calculated. As the bandwidth of the used IF-filter with a Gaussian characteristic was $120 \mathrm{kHz}$, the resulting powers were normalized to an IF-bandwidth of $1 \mathrm{~Hz}$, using

$P_{\mathrm{NF}, \mathrm{AV}}=P_{N} / B_{\mathrm{ENB}, \mathrm{IF}}$,

where $P_{\mathrm{NF}, \mathrm{AV}}$ is the average power of the system's noise floor, and $B_{\mathrm{ENB}, \mathrm{IF}}$ is the equivalent noise bandwidth of the IF-filter. The results are given in Table 1 and show that the system noise floor power spectral density from $6-18 \mathrm{GHz}$ stays below $-160 \mathrm{dBm} \mathrm{Hz}^{-1}$.

Measurements of the radiated emission of electronic consumer devices and electric household appliances were performed in a full anechoic chamber. A broadband quad-ridged horn antenna with a bandwidth from $1.7-20 \mathrm{GHz}$ was used (Datasheet RF-Spin QRH20, 2010). To compensate for cable losses and to give the electric field strength of the EMI, the corresponding transducer factors and the antenna factor were applied.

Figure 10 shows a spectrogram of the WI-FI data between two modern smartphones. The two phones formed a wire-

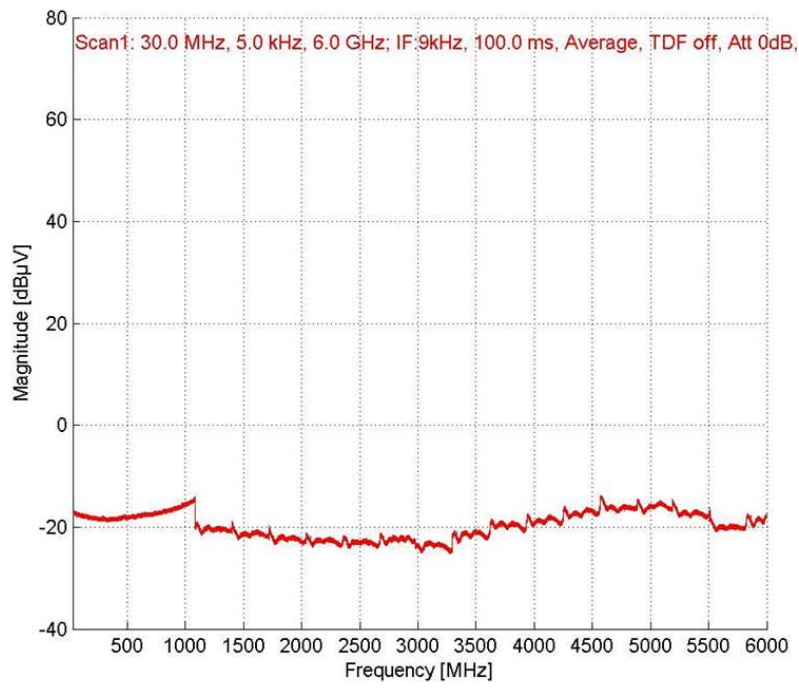

Fig. 9. System noise floor from $30 \mathrm{MHz}$ to $6 \mathrm{GHz}$.

Table 1. System noise floor from $6 \mathrm{GHz}$ to $18 \mathrm{GHz}$.

\begin{tabular}{cc}
\hline Frequency/MHz & Power spectral density/dBm Hz \\
\hline 6000 & -164.5 \\
7000 & -164.4 \\
8000 & -164.7 \\
9000 & -163.1 \\
10000 & -163.2 \\
11000 & -163.4 \\
12000 & -162.8 \\
13000 & -162.8 \\
14000 & -162.9 \\
15000 & -162.6 \\
16000 & -161.4 \\
17000 & -161.4 \\
18000 & -160.7 \\
\hline
\end{tabular}

less network and files were transmitted via the file-transferprotocol (FTP). The antenna was placed in a distance of $3 \mathrm{~m}$ to the test subjects. The real-time spectrogram allows for the examination of the time-behaviour. While the WI-FI network was idle for the most part, the transmission of a file, lasting from $2 \mathrm{~s}$ to $6 \mathrm{~s}$, is visible.

For the measurement shown in Fig. 11, a microwave oven was placed in a distance of $3 \mathrm{~m}$ to the antenna. The spectrum shows the magnetron's fundamental at around $2.45 \mathrm{GHz}$ and several higher harmonics up to $18 \mathrm{GHz}$. The scan time using an IF-filter bandwidth of $9 \mathrm{kHz}$ and a frequency resolution of $50 \mathrm{kHz}$ took around $120 \mathrm{~s}$.

The magnetron's output frequency is non-stationary. The time-domain system's real-time capability allows for the examination of the time-behaviour of the magnetron's 6th harmonic. In Fig. 12, the spectrogram shows the time-behaviour 


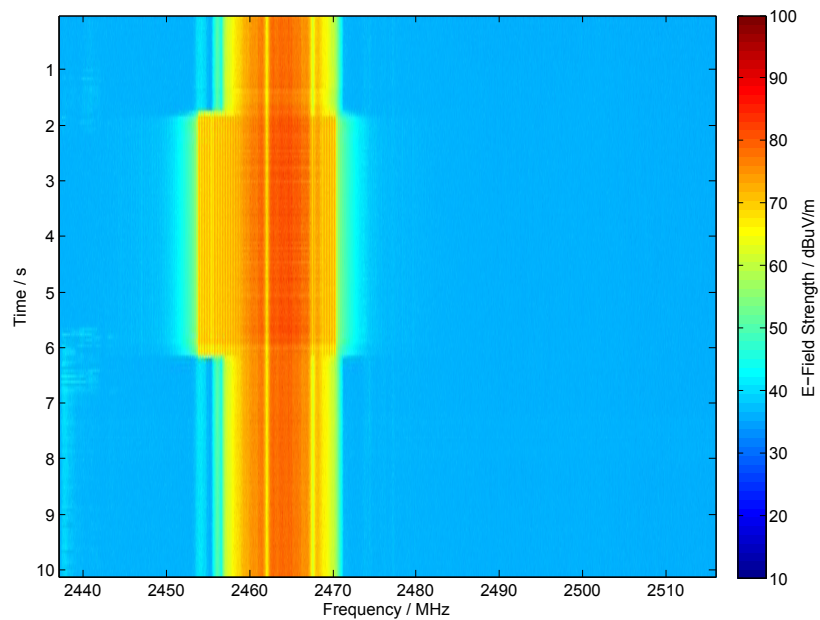

Fig. 10. Spectrogram of WI-FI communication between two mobile phones.

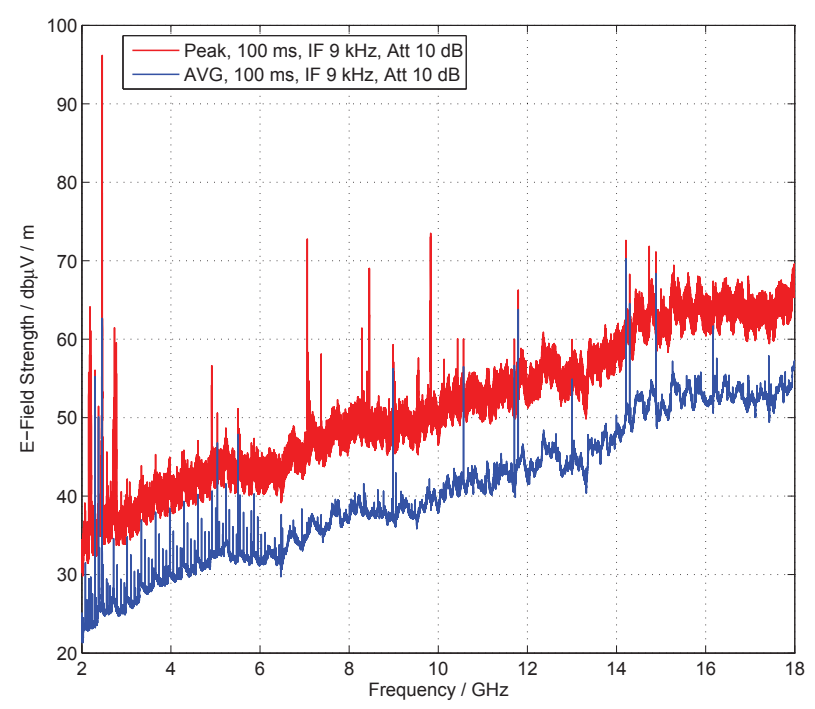

Fig. 11. Emission spectrum of a microwave oven.

of the magnetron's 6th harmonic over a $20 \mathrm{~s}$ time-period. In a medium power setting, the magnetron turns on at around $2 \mathrm{~s}$ in time and turns off at $8 \mathrm{~s}$. The change in frequency of the free-running oscillator is visible. The high sensitivity of the system and the real-time operation with an IF-bandwidth of $9 \mathrm{kHz}$ enables the measurement of the 6th harmonic, which is located around $20 \mathrm{~dB}$ above the system noise floor.

\section{Conclusions}

A highly sensitive time-domain EMI measurement system for the frequency range from $9 \mathrm{kHz}$ to $18 \mathrm{GHz}$ was presented. The system provides for EMI measurements in CISPR Band $\mathrm{E}$ in accordance to the requirements stated in

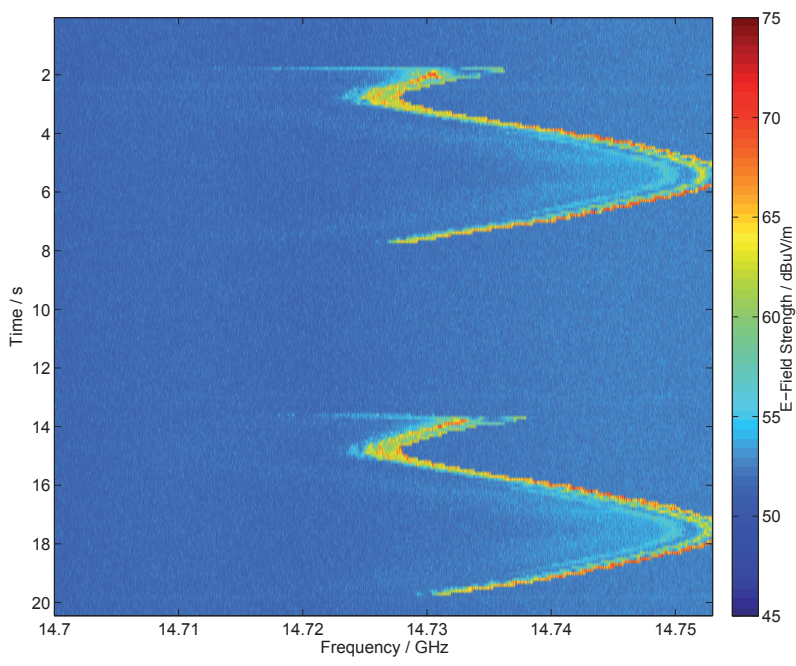

Fig. 12. Spectrogram of the 6th harmonic of a microwave oven.

CISPR 16-1-1. In comparison to traditional EMI-receivers, scan times can be decreased by several orders of magnitude. The high sensitivity, due to a low system noise floor of $6-8 \mathrm{~dB}$, allows for the broadband characterisation of narrowband interference near the noise floor. Measurements of electric household appliances and consumer electronic devices were presented, including measurements of the subjects time-behaviour via the spectrogram.

Acknowledgements. The authors would like to thank the Bayerische Forschungsstiftung and GAUSS Instruments $\mathrm{GmbH}, \mathrm{Mu}-$ nich, Germany for funding this project and the EMV GmbH, Taufkirchen, Germany for supplying the antenna for the measurements. The authors are indebted to Stephan Braun and Arnd Frech from GAUSS Instruments $\mathrm{GmbH}$ for numerous helpful discussions.

\section{References}

Braun, S. and Russer, P.: A low-noise multiresolution high-dynamic ultra-broad-band time-domain EMI Measurement System, IEEE Transactions on Microwave Theory and Techniques, 53, 33543363, 2005.

Braun, S., Hoffmann, C., and Russer, P.: A Realtime Time-domain EMI Measurement System for Measurements above $1 \mathrm{GHz}$, IEEE EMC Society Symposium on Electromagnetic Compatibility, Austin, USA, 2009.

Braun, S., Hoffmann, C., and Russer, P.: Emissionsmessung im Zeitbereich oberhalb $1 \mathrm{GHz}$, EMV 2010, Düsseldorf, Germany, 2010.

CISPR 16-1-1, Ed. 3.1 Am. 1: Specification for radio disturbance and immunity measuring apparatus and methods Part 1-1: Radio disturbance and immunity measuring apparatus - Measuring apparatus, International Electrotechnical Commission, 2010.

Datasheet RF Spin: Broadband Quad-Ridged Horn Antenna QRH20, Data Sheet, 2010.

Davenport, W. B. and Root, D. L.: An Introduction to the Theory of Random Signals and Noise, John Wiley \& Sons, 1987. 
Hagenhaus, K.: Die Messung von Funkstörungen, Elektrotechnische Zeitschrift, 63, 182-187, 1942.

Hoffmann, C., Braun, S., and Russer, P.: A Broadband TimeDomain EMI Measurement System for Measurements up to $18 \mathrm{GHz}$, EMC Europe 2010, Wroclaw, Poland, 34-37, 2010.

Maas, S. A.: Microwave Mixers, Artech House, 1993.
Oppenheim, A. V. and Schafer, R. W.: Discrete-Time Signal Processing, 3rd Edition, Pearson Prentice Hall, 2009.

Sze, S. M.: Modern Semiconductor Device Physics, John Wiley \& Sons, 1988.

Vendelin, G. D., Pavio, A. M., and Rohde, U. L.: Microwave Circuit Design using Linear and Nonlinear Techniques, second edition, John Wiley \& Sons, 2005. 